\title{
The Korteweg-de Vries Equation for Wave Propagation in an Infinitely Long Thin Walled Circular Cylinder Obtained via the Lagrangian Method
}

\author{
K. Murawski \\ Department of Physics, Akademicka 13, 20-033 Lublin, Poland
}

Z. Naturforsch. 40 a, 955-956 (1985); received April 18, 1985

On the basis of the Lagrangian method a nonlinear wave theory is developed to obtain the Korteweg-de Vries equation for the incompressible, one-dimensional, weak-nonlinear, and dispersive motion of fluid in an infinitely long circular cylinder (with thin walls of elastic rings). This equation has first been obtained by Lamb [1] via the reductive Taniuti-Wei's method [2].

The nonlinear partial differential equations which govern wave phenomena in fluid filled tubes have resisted analysis for many years. It has been shown that in the presence of dissipation the fundamental set of equations for the irrotational motion of waves can be reduced to Burgers equation [3-5]. In 1969 Johnson introduced the so-called Korteweg-de VriesBurgers equation for a wave propagation on an elastic tube filled with a viscous fluid, which may be regarded as a simple model of an artery [6]. Also wave propagation and shock formation in nonlinear elastic and viscoelastic fluid filled tubes has been discussed for a Mooney-Rivilin material [7]. Some time ago, Lamb [1] has used the reductive TaniutiWei's method to obtain the Korteweg-de Vries equation for the one-dimensional, irrotational, weak-nonlinear, and incompressible motion of fluid that is confined within an infinitely long circular cylinder (with walls of elastic rings). For this case the governing equations are the conservation of mass equation given by

$$
A_{t}+(A V)_{x}=0 \text {, }
$$

Euler's equation,

$$
V_{t}+V V_{x}+\frac{1}{\varrho_{0}} p_{x}=0,
$$

and Newton's equation

$$
A_{t t}+\frac{E}{\varrho_{m} a^{2}}\left(A-\pi a^{2}\right)-\frac{2 \pi a}{\varrho_{m} h} p=0 .
$$

The symbols used have the following meanings: $A$ section field, $V$ velocity of fluid, $\varrho_{0}$ density of fluid,

Reprint requests to K. Murawski, Department of Physics, Akademicka 13, 20-033 Lublin, Poland. $p$ pressure of fluid, $E$ Young's modulus, $\varrho_{m}$ density of the tube material, $h$ thickness of a tube, $a$ radius of a tube at the undisturbed uniform state. The subscripts $x$ and $t$ indicate partial differentiation.

Introducing new coordinates as follows

$$
\begin{aligned}
& A \rightarrow\left(\pi a^{2}\right)^{-1} A, p \rightarrow p_{0}^{-1} p, \quad t \rightarrow T^{-1} t, \\
& x \rightarrow L^{-1} x, \quad V \rightarrow \frac{T}{L} V, \quad p_{0}=\frac{E h}{2 a}, \\
& T^{2}=\frac{\varrho_{m} a^{2}}{E}, \quad L^{2}=\frac{\varrho_{m} a h}{2 \varrho_{0}},
\end{aligned}
$$

dimensionless equations are obtained, i.e.,

$$
\begin{aligned}
& A_{t}+(A V)_{x}=0, \\
& V_{t}+V V_{x}+p_{x}=0, \\
& A_{t t}+A-p=1 .
\end{aligned}
$$

Application of the reductive Taniuti-Wei's method to $(5)-(7)$ corresponds to the usual way for constructing the Korteweg-de Vries equation.

However, our way is rather different. Using a Lagrangian for the fundamental set of equations $(5)-(7)$, we derive the Korteweg-de Vries equation via the $\varepsilon$ expansion method for the Lagrangian only. This method has been presented in [8].

Without loss of generality we take $V$ to have the form

$$
V=\Psi_{x}
$$

Substituting this definition and (7) into (5) and (6), we obtain

$$
\begin{aligned}
& A_{t}+\left(A \Psi_{x}\right)_{x}=0 \\
& \Psi_{t}+\frac{1}{2} \Psi_{x}^{2}+A_{t t}+A=1
\end{aligned}
$$


A Lagrangian for the above set of equations can be found and written as follows:

$$
L=\frac{1}{2} A \Psi_{x}^{2}+A \Psi_{t}-\frac{1}{2} A_{t}^{2}+\frac{1}{2} A^{2}-A+\frac{1}{2} .
$$

We introduce the stretched coordinates

$$
\begin{aligned}
Y & =\varepsilon^{1 / 2}(x+t), \\
\tau & =\varepsilon^{3 / 2} t
\end{aligned}
$$

together with the following ordering for $A, \Psi$, and $L$

$$
\begin{aligned}
& A=1+\varepsilon A_{1}+\ldots, \\
& \Psi=\varepsilon^{1 / 2} \Psi_{1}+\varepsilon^{3 / 2} \Psi_{2}+\ldots, \\
& L=L_{0}+\varepsilon L_{1}+\ldots .
\end{aligned}
$$

All quantities are considered to be functions of $Y$ and $\tau$ only. In the new coordinates the Lagrangian becomes

$$
\begin{aligned}
L= & \frac{1}{2} \varepsilon A \Psi_{Y}^{2}+\varepsilon^{1 / 2} A \Psi_{Y}+\varepsilon^{3 / 2} A \Psi_{\tau}+\frac{1}{2} A^{2}-A+\frac{1}{2} \\
& -\frac{1}{2}\left(\varepsilon A_{Y}^{2}+2 \varepsilon^{2} A_{Y} A_{\tau}+\varepsilon^{3} A_{\tau}^{2}\right) .
\end{aligned}
$$

Inserting expressions (13) into the Lagrangian and equating coefficients of the same powers of $\varepsilon^{0}, \varepsilon^{1}, \varepsilon^{2}$, and $\varepsilon^{3}$, we obtain

$$
\begin{aligned}
& L_{0}=0, \quad L_{1}=\Psi_{1 Y}, \\
& L_{2}=\frac{1}{2} \Psi_{1 Y}^{2}+A_{1} \Psi_{1 Y}+\Psi_{1 \tau}+\frac{1}{2} A_{1}^{2}, \\
& L_{3}=\frac{1}{2} A_{1} \Psi_{1 Y}^{2}+A_{1} \Psi_{1 \tau}-\frac{1}{2} A_{1 Y}^{2},
\end{aligned}
$$

[1] G. L. Lamb, Jr., Elements of Soliton Theory, John Wiley, New York 1980.

[2] T. Taniuti and C. C. Wei, J. Phys. Soc. Japan 24, 941 (1968).

[3] P. L. Bhatnagar, Nonlinear Waves in one-dimensional Dispersive Systems, Claredon Press, Oxford 1979.

[4] K. Murawski, Acta Phys. Pol. A69/1, 1986, to be published. respectively. So the Euler-Lagrange equation for $L_{2}$ leads to the expression

$$
\frac{\delta L_{2}}{\delta A_{1}}=\Psi_{1 Y}+A_{1}=0 .
$$

Introducing this equation into $L_{3}$, the EulerLagrange equation takes the following form:

$$
\frac{\delta L_{3}}{\delta \Psi_{1}}=2 \Psi_{1 \tau Y}+\frac{3}{2}\left(\Psi_{1 Y}^{2}\right)_{Y}-\Psi_{1 Y Y Y Y}=0
$$

Bearing transformation (8) in mind, the Kortewegde Vries equation can be obtained,

$$
V_{1 \tau}+\frac{3}{2} V_{1} V_{1 Y}-\frac{1}{2} V_{1 Y Y Y}=0 \text {. }
$$

Based on the rigorous method developed in the Lagrangian perturbation theory, we have derived the Kortweg-de Vries equation as a first-order of approximation which describes the weak-nonlinear propagation of waves in an infinite thin walled circular cylinder. It differs from the corresponding equation as obtained by Lamb by the sign of the last term. But since we have introduced a different sign for the time $t$ in the coordinate $Y$, see the formula $(12 \mathrm{a})$, our result is not at variance with the one of [1].

The author would like to thank Dr. Ernst F. Hefter for his valuable remarks.

[5] K. Murawski, Z. Naturforsch., to be published.

[6] R. S. Johnson, Doctoral thesis, Univ. London, London 1969.

[7] R. J. Tait and T. B. Moodie, Wave Motion 6, 197 (1984).

[8] E. Infeld, Acta Phys. Pol. A60, 623 (1981). 